\title{
Capsule Commentary on Thorp et al., Implementation of 2011 Duty Hours Regulations Through a Workload Reduction Strategy and Impact on Residency Training
}

\author{
Michael J. Rosenblum, MD, FACP \\ Baystate Medical Center, University of Massachusetts Medical School, Springfield, MA, USA.
}

$\mathrm{J}$ Gen Intern Med 31(12): 1506

DOI: $10.1007 / \mathrm{s} 11606-016-3863-3$

(c) Society of General Internal Medicine 2016

$\mathrm{T}$ horp et al. ${ }^{1}$ investigated the impact of workload reduction on PGY-1 resident training experiences and care quality measures. For 30 PGY-1 residents in the intervention group, who began training between July 1, 2011, and June 30, 2013, the number of inpatient encounters was similar to the 28 PGY1 in the control cohort (7/1/2009-6/30/2011). The exposed group had $15 \%$ fewer admissions and $13 \%$ more outpatient patient visits. The Internal Medicine In-Training Examination (IM-ITE) was used as a surrogate for medical knowledge, with no significant differences between groups. Care quality markers were unchanged or improved. The authors conclude that reducing admission volume does not have a negative impact on resident education or on a number of clinical outcome measures.

The intervention included a reduction in the number of admissions, the development of a night and day team wards model, and modifications in the medical intensive care unit (MICU)/intermediate care (IMC) schedules. Twelve days of electives were discontinued, and 30 fewer days of critical care were offset by increases in ward and outpatient time.

Determining appropriate clinical and learning experiences are crucial concerns for the educational community. The FIRST trial revealed no impact on clinical or educational outcomes from flexible vs. restrictive surgical duty hour regulations. ${ }^{2}$ The iCOMPARE study comprising 63 IM residency programs will add to our understanding of the educational and clinical impact of duty hours. ${ }^{3}$

In addition to the single-center study design, interpretation of the findings may be confounded by institutional modifications impacting patient outcome measures. In our era of the Affordable Care Act (ACA) and the primary care crisis, ${ }^{4}$ addressing inpatient and ambulatory training disparities is fertile ground for research and innovation. This study helps us recognize the impact of workload reduction on resident experience, but does not analyze ultimate career determinations.

A stakeholder conversation to determine how graduate medical education (GME) (and undergraduate medical education (UME)) can improve alignment with national and community needs would be a stepping stone toward refinement of our training models. In the future, workload reduction and other innovative strategies can help transform residency training into a learner-specific, and ultimately career-specific (learner-centered education), direction.

Corresponding Author: Michael J. Rosenblum, MD, FACP; Baystate Medical CenterUniversity of Massachusetts Medical School, Springfield, MA, USA (e-mail: Michael.rosenblum@baystatehealth.org).

Compliance with Ethical Standards:

Conflict of Interest: The author declares that he has no conflict of interest.

\section{REFERENCES}

1. Thorp J, Dattalo M, Ghanem KG, Christmas C. Implementation of 2011 duty hours regulations through a workload reduction strategy and impact on residency training. J Gen Intern Med. 2016. doi:10.1007/s11606-0163840-x.

2. Bilimoria KY, Chung JW, Hedges LV, et al. National cluster-randomized trial of duty-hour flexibility in surgical training. N Engl J Med 2016;374:713-727. doi:10.1056/NEJMoa1515724

3. iCOMPARE information https://clinicaltrials.gov/ct2/show/NCT02274818 http://www.jhcct.org/icompare/default.asp

4. Long T, Chaiyachati $\mathbf{K}$, Bosu O, et al. Why aren't more primary care residents going into primary care? A qualitative study. J Gen Intern Med. 2016. doi:10.1007/s11606-016-3825-9.

Published online October 19, 2016 